\title{
Temperature regulates splicing efficiency of the cold-inducible RNA-binding protein gene Cirbp
}

\author{
Ivana Gotic, ${ }^{1}$ Saeed Omidi, ${ }^{2}$ Fabienne Fleury-Olela, ${ }^{1}$ Nacho Molina, ${ }^{2,3}$ Felix Naef, ${ }^{2}$ and Ueli Schibler ${ }^{1}$ \\ ${ }^{1}$ Department of Molecular Biology, University of Geneva, CH-1211 Geneva 4, Switzerland; ${ }^{2}$ The Institute of Bioengineering, \\ School of Life Sciences, Ecole Polytechnique Fédérale de Lausanne, Swiss Institute of Bioinformatics, CH-1015 \\ Lausanne, Switzerland
}

In mammals, body temperature fluctuates diurnally around a mean value of $36^{\circ} \mathrm{C}-37^{\circ} \mathrm{C}$. Despite the small differences between minimal and maximal values, body temperature rhythms can drive robust cycles in gene expression in cultured cells and, likely, animals. Here we studied the mechanisms responsible for the temperaturedependent expression of cold-inducible RNA-binding protein (CIRBP). In NIH3T3 fibroblasts exposed to simulated mouse body temperature cycles, Cirbp mRNA oscillates about threefold in abundance, as it does in mouse livers. This daily mRNA accumulation cycle is directly controlled by temperature oscillations and does not depend on the cells' circadian clocks. Here we show that the temperature-dependent accumulation of Cirbp mRNA is controlled primarily by the regulation of splicing efficiency, defined as the fraction of Cirbp pre-mRNA processed into mature mRNA. As revealed by genome-wide "approach to steady-state" kinetics, this post-transcriptional mechanism is widespread in the temperature-dependent control of gene expression.

[Keywords: Cirbp; splicing efficiency; temperature; circadian rhythms]

Supplemental material is available for this article.

Received July 12, 2016; revised version accepted August 19, 2016.

Temperature is a fundamental physical parameter that influences metabolism in all organisms by modulating the rate of biochemical reactions. Endothermic organisms like mammals have acquired special thermoregulatory adaptation mechanisms in order to decrease their dependence on environmental temperature. These mechanisms maintain core body temperature (CBT) within a favorable range, allowing efficient metabolic activity throughout diurnal and seasonal cycles (for review, see Tattersall 2012).

Although regulated around a species-specific set point, mammalian CBT shows diurnal oscillations of $\sim 0.4^{\circ} \mathrm{C}-$ $6.0^{\circ} \mathrm{C}$ (for review, see Refinetti 2010). These regular oscillations are generated under the control of the circadian timekeeping system, which is composed of a master pacemaker in the brain's suprachiasmatic nucleus (SCN) and subsidiary peripheral clocks in nearly all other cells of the body (Dibner et al. 2010). Although peripheral circadian oscillators are self-sustained and cell-autonomous,

\footnotetext{
${ }^{3}$ Present address: Institute of Genetics and Molecular and Cellular Biology, Centre National de la Recherche Scientifique, Institut National de la Santé et de la Recherche Médicale, University of Strasbourg, 67400 Illkirch, France

Corresponding author: ueli.schibler@unige.ch

Article is online at http://www.genesdev.org/cgi/doi/10.1101/gad.287094. 116. Freely available online through the Genes \& Development Open Access option.
}

they must be synchronized by the SCN and/or environmental Zeitgebers (timing cues), such as light-dark cycles, in order to maintain phase coherence and rhythmic physiology in the body. Without a functional SCN, laboratory rodents kept in constant darkness lose virtually all overt rhythms in behavior and physiology, including CBT cycles (Refinetti et al. 1994).

While the period length $(\tau)$ of circadian oscillations is remarkably resilient to temperature variations, a phenomenon called temperature compensation, the phase is exquisitely sensitive to temperature perturbations, in particular in peripheral cells. In fact, in cultured fibroblasts and tissue explants, the phase of circadian clock gene expression can be perfectly synchronized by simulated CBT cycles (Brown et al. 2002; Buhr et al. 2010; Saini et al. 2012).

In intact animals, the systemic synchronization cues governed by the SCN and/or environment also comprise feeding-fasting behavior and blood-borne factors (for review, see Schibler et al. 2015). Owing to this complexity, it is challenging to assess to what extent CBT rhythms contribute to diurnal gene expression and the

(C) 2016 Gotic et al. This article, published in Genes \& Development, is available under a Creative Commons License (Attribution-NonCommercial 4.0 International), as described at http://creativecommons.org/licenses/by-nc/4.0/. 
synchronization of peripheral clocks in vivo. Nonetheless, experiments with mice harboring inactive hepatocyte clocks revealed robust diurnal accumulation cycles for a number of heat- and cold-inducible transcripts. The peak expression of these transcripts at times when CBT was maximal and minimal, respectively, suggests that they are likely driven directly by body temperature oscillations (Kornmann et al. 2007).

The up-regulation of many heat-inducible genes is mediated by transcriptional mechanisms depending on heatshock transcription factors, mostly HSF1, whose monomers are sequestered into inert cytosolic protein complexes with HSP90 and other proteins at a lower CBT. Upon temperature elevation, HSF1 monomers get released, trimerize, translocate into the nucleus, and bind to heatshock response elements (HSEs) within promoter and enhancer sequences of their target genes. The circadian core clock gene Per2 is among these targets, possibly explaining the contribution of HSF1 to the synchronization of circadian gene expression by simulated CBT rhythms (Reinke et al. 2008; Buhr et al. 2010; Tamaru et al. 2011; Saini et al. 2012).

In contrast to the mechanisms governing heat-induced gene expression, those involved in cold-induced gene expression are still largely elusive. Cirbp, encoding the cold-inducible RNA-binding protein CIRBP (also abbreviated as CIRP), is among the proteins whose accumulation displays diurnal oscillations that are likely driven by CBT rhythms (Nishiyama et al. 1997, 1998; Kornmann et al. 2007; Morf et al. 2012). CIRBP was originally described as a human UV stress-induced factor, then named A18 hnRNP, involved in the cellular genotoxic stress response (Fornace et al. 1988; Yang and Carrier 2001). Its temperature-dependent expression became apparent during a PCR-based search for RNA-binding domain-containing proteins (RBPs) present in mouse testes (Nishiyama et al. 1997, 1998). CIRBP appears to be an important modulator of proliferation in cellular stress conditions, including cancer, where it is thought to exert an activating or inhibitory role depending on the cell type and the transformation state of the tumor (Hamid et al. 2003; Masuda et al. 2012; Ren et al. 2014; Sakurai et al. 2015; Wang et al. 2015).

CIRBP is thought to protect bound target RNAs from degradation, enhance their export and translation in the cytoplasm (Yang and Carrier 2001; Morf et al. 2012), and regulate the choice of alternative polyadenylation sites (Liu et al. 2013). The role of CIRBP in circadian gene expression was discovered by loss-of-function experiments, which demonstrated its requirement for robust circadian gene expression in cultured NIH3T3 cells and mouse embryonic fibroblasts (Morf et al. 2012).

Here we elucidate the molecular mechanisms underpinning temperature-mediated Cirbp expression in NIH3T3 fibroblasts. We show that pre-mRNA splicing efficiency is a critical parameter accounting, to a large extent, for the increased Cirbp mRNA accumulation at reduced temperatures. Moreover, the recording of genomewide "approach to steady-state" (ATSS) kinetics of RNA transcript accumulation indicates that this mechanism is widespread in the control of temperature-dependent gene expression.

\section{Results \\ Mild cold exposure increases the expression of a single Cirbp mRNA isoform without affecting its pre-mRNA levels}

In various mouse tissues, Cirbp mRNA levels follow a diurnal pattern, reaching zenith and nadir values when body temperature is minimal and maximal, respectively (Supplemental Fig. S1A,B). Since local circadian oscillators are not required for these oscillations in animals (Kornmann et al. 2007) and since Cirbp mRNA accumulation is known to be temperature-dependent, we considered rhythmic Cirbp expression to be likely driven directly by CBT cycles. Indeed, simulated CBT rhythms are sufficient to elicit cyclic Cirbp mRNA and protein expression in cultured NIH3T3 fibroblasts (Morf et al. 2012), and we therefore used these cells to study the underpinning mechanisms. In contrast to mouse livers, this model system allowed us to identify the impact of temperature in the absence of potential confounding effects caused by circulating hormones and metabolites.

Previous studies have shown that mild cold exposure $\left(32^{\circ} \mathrm{C}\right)$ increases the concentration of Cirbp mRNA in cultured cells (Nishiyama et al. 1997). To examine whether the level of Cirbp pre-mRNA transcripts was also changed during cold exposure, we performed RNase protection assays (RPAs). By using an RNA probe that protects the intron 6-exon 7 boundary region of Cirbp transcripts from RNase A/T1 digestion, we were able to simultaneously quantify the levels of Cirbp pre-mRNA and mRNA species within a particular sample (Fig. 1A). As shown in Figure 1, B and C, cold exposure caused an increase in Cirbp mRNA but did not affect the level of its precursors. A similar conclusion was reached for diurnal Cirbp mRNA and pre-mRNA accumulation in mouse livers when we analyzed published RNA sequencing (RNA-seq) data (Atger et al. 2015). While Cirbp mRNA oscillated in abundance with the expected phase, Cirbp pre-mRNA levels remained nearly constant throughout the day (Supplemental Fig. S1C).

Next, we asked whether different temperatures induced the expression of specific Cirbp mRNA isoforms by either the use of different transcription start sites (TSSs), alternative splicing, and/or the use of different polyadenylation sites. To this end, we performed Northern blot experiments and analyzed the composition of transcripts at different temperatures by sequencing $5^{\prime}$ and $3^{\prime}$ products obtained in RACE (rapid amplification of cDNA ends) reactions. The Northern blot analysis revealed a single band corresponding to 1400 nucleotides for Cirbp mRNA irrespective of whether the RNA was extracted from cytoplasm or nuclei (Fig. 1D). As demonstrated by RPA and RACE analyses, the lengths (Supplemental Fig. S1D-F) and sequences (data not shown) of Cirbp mRNA 5' and $3^{\prime}$ regions were indistinguishable at different temperatures. 


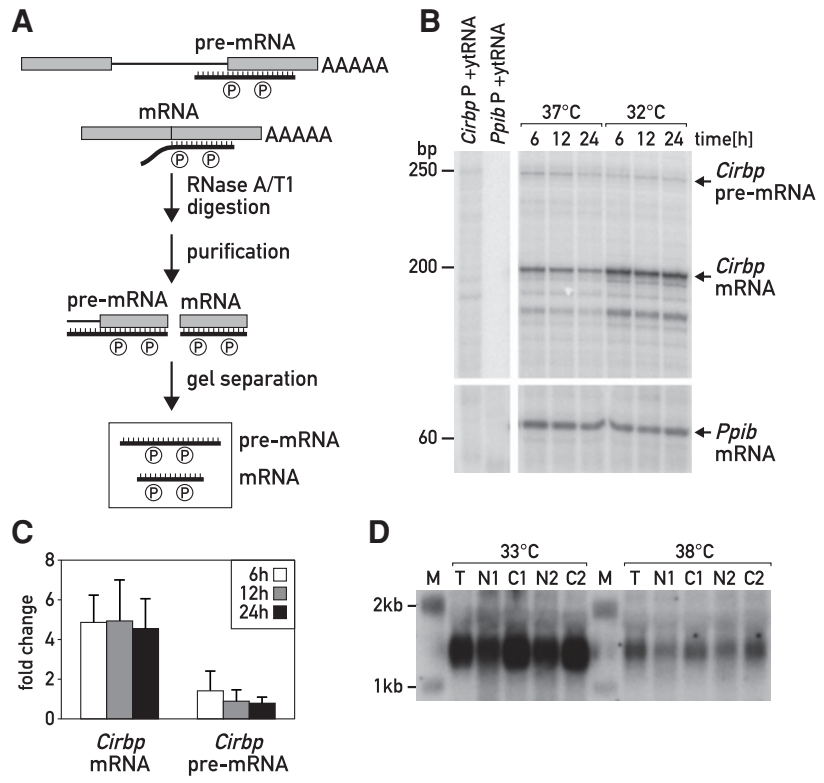

Figure 1. Mild cold exposure increases the expression of a single Cirbp mRNA isoform without affecting its pre-mRNA levels in NIH3T3 cells. (A) Schematic representation of the RPA. A genespecific radioactive RNA probe protects the intron-exon border of the unspliced transcripts and the remaining exon region of the spliced mRNA from RNaseA/T1-mediated degradation. The protected RNA fragments were size-fractionated on $12 \%$ ureapolyacrylamide sequencing gels and quantified by phosphorimaging. $(B)$ RPA with a probe mix protecting the Cirbp intron 6-exon 7 border ( 50 base pairs [bp] of intron 6 and $200 \mathrm{bp}$ of exon 7) and the first 60 bp of Ppib exon 6 in RNA samples from cells incubated for various time periods at $37^{\circ} \mathrm{C}$ and $32^{\circ} \mathrm{C}$. (P) Probe; (ytRNA) yeast tRNA. (C). Quantification of Cirbp mRNA and pre-mRNA levels by RPA $(B)$, represented as fold changes at the indicated time intervals after shifting the cells from $37^{\circ} \mathrm{C}$ to $32^{\circ} \mathrm{C}$. The Cirbp transcript signals were normalized to $P$ pib transcript signals (see $B$ ). The data (average value \pm standard deviations [SDs]) from three biological replicates are given. $(D)$ Northern blot assay for Cirbp transcripts. The radioactive RNA probe was complementary to the full-length Cirbp mRNA. RNA was isolated from either total cell extracts $(T)$, purified nuclei $(N)$, or cytoplasm $(C)$. (M) RNA size markers.

Temperature-dependent Cirbp mRNA accumulation is regulated by a post-transcriptional mechanism

Since Cirbp pre-mRNA levels were found to be rather constant at different temperatures, we considered that Cirbp transcription might also be temperature-independent. To test this hypothesis, we performed a chromatin immunoprecipitation (ChIP) assay with an antibody directed against RNA polymerase II subunit b (POLR2B). Specifically, we quantified the levels of immunoprecipitated DNA fragments encompassing the Cirbp promoter or gene body regions in cells incubated for $6 \mathrm{~h}$ at $33^{\circ} \mathrm{C}$ or $38^{\circ} \mathrm{C}$ by quantitative real-time PCR (RT-qPCR). Our results revealed a prevalent presence of POLR2B at the Cirbp promoter, which was similar in both conditions, and barely detectable POLR2B levels at the exon 6-intron 6 border-spanning region of the gene (Fig. 2A,B). The dra- matically higher density of POLR2B within the promoter-proximal region compared with the rest of the gene body probably implies a pausing of the polymerase within the 5'-proximal 200 base pairs (bp). It could be argued that the release of polymerase into the gene body and hence transcription efficiency could be temperature-dependent. However, this mechanism would not be compatible with the observation that pre-mRNA species encompassing downstream Cirbp regions do not vary in a temperaturedependent manner (Fig. 1B,C).

If Cirbp activity was regulated by post-transcriptional mechanisms, a temperature-insensitive promoter should still drive oscillating Cirbp expression in cells exposed to simulated CBT cycles. To examine this conjecture, we engineered a Cirbp-luciferase fusion gene in which the Cirbp promoter was replaced by the human cytomegalovirus (CMV) promoter. The activity of the commonly used Photinus phyralis firefly luciferase is considerably higher at reduced temperatures. This effect is attributed to reduced protein activity and a so-called red shift in the luminescence at higher temperatures (for review, see Koksharov and Ugarova 2012). Therefore, we used the more temperature-tolerant mutant Luciola mingrelica firefly luciferase (LMLucR) (Koksharov and Ugarova 2011) for the construction of the Cirbp-luciferase reporter gene. This enabled us to detect relatively modest amplitudes in temperature-dependent reporter gene expression in phase with the intrinsic temperature sensitivity of the luciferase protein.

For the construction of the reporter gene dubbed gCirbp-LMLucR, the entire genomic Cirbp-encoding DNA region of $3.8 \mathrm{~kb}$ was cloned downstream from the CMV promoter and fused in-frame the with LMLucR gene. NIH3T3 cells were transiently transfected with the reporter construct and exposed to simulated CBT cycles, and the resulting bioluminescence was recorded in real-time during the following 6 d. As shown in Figure $2 \mathrm{C}$, even under the control of a constitutively active promoter, the gCirbp-LMLucR construct exhibited temperature-dependent oscillations with the expected phase. This suggested the presence of a temperature-responsive element within the Cirbp pre-mRNA-encoding region and its independence of the Cirbp promoter. The low-amplitude bioluminescence oscillations detected in cells transfected with the control CMV-LMLucR construct (Fig. 2C) were not a reflection of transgene mRNA levels (Supplemental Figs. S2A, S3G). Likely, these were caused by residual temperature effects on the aforementioned luciferase protein characteristics.

\section{Changes in mRNA stability contribute moderately to Cirbp temperature sensitivity}

Having excluded transcription as the major mechanism for temperature-responsive Cirbp expression, we examined whether the stability of Cirbp mRNA was temperature-dependent. Generally, mRNA half-lives are estimated by measuring the levels of specific transcripts at increasing time intervals after the addition of transcription-blocking drugs or, alternatively, after metabolic 


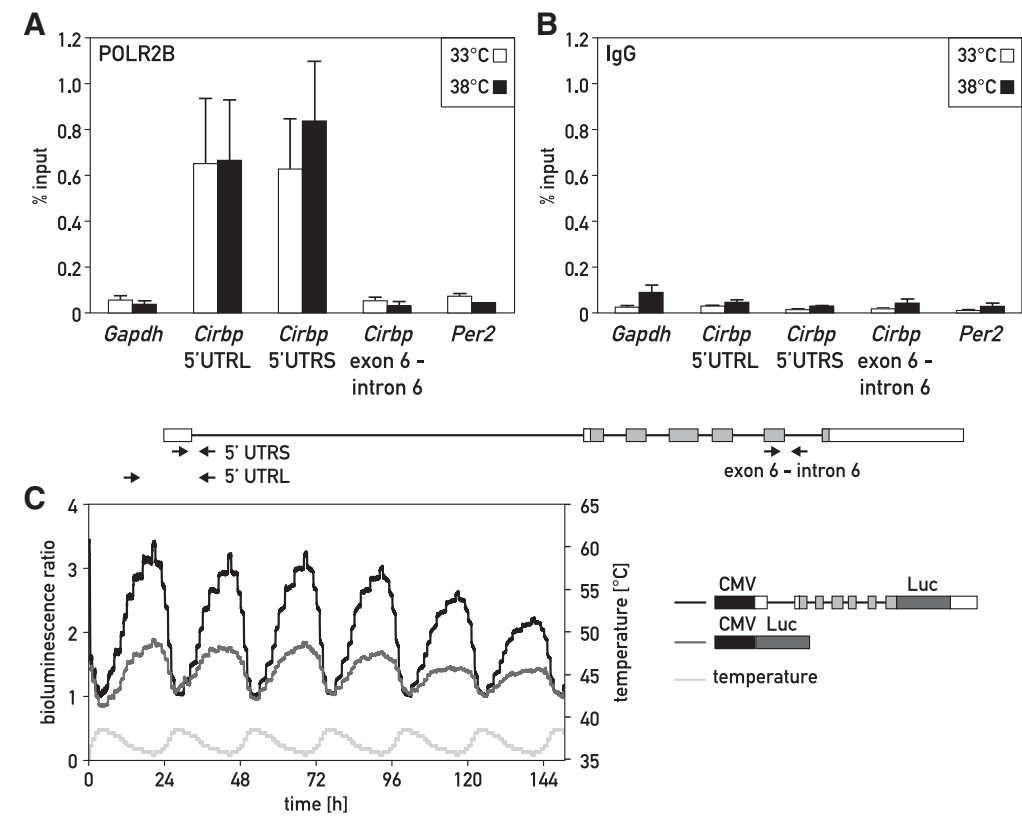

Figure 2. Temperature-dependent Cirbp mRNA accumulation is regulated by a post-transcriptional mechanism. (A) POLR2B ChIP in cells incubated for $6 \mathrm{~h}$ at $33^{\circ} \mathrm{C}$ or $38^{\circ} \mathrm{C}$. (B) IgG control ChIP. The Cirbp gene structure and the qPCR-amplified regions are depicted below the graphs. Data are presented as percentage of input DNA. $n=3-5$ biological replicates. The bars represent SDs. (C) Cytomegalovirus (CMV)gCirp-LMLucR reporter assay in cells exposed to simulated CBT cycles of $35.5^{\circ} \mathrm{C}-38.5^{\circ} \mathrm{C}$ for $6 \mathrm{~d}$. Reporter construct schemes are depicted at the right, with Cirbp untranslated region (UTR)-encoding exons illustrated in white and protein-encoding exons shown in light gray. A representative recording of $>10$ experiments is shown. Data were normalized to the baseline curve explained in Supplemental Figure S2B. labeling and isolation of newly synthesized RNA transcripts (for review, see Bensaude 2011). However, the RNA polymerase inhibitors and labeling components used by these methods can alter mRNA metabolism in cells (Fraschini et al. 2005), and their uptake, pool sizes, and/or activity rates may be different at different temperatures.

To overcome these limitations, we used a noninvasive method known as ATSS (Greenberg 1972; Harrold et al. 1991) to estimate the half-life of Cirbp mRNA. The power of the ATSS method lies in its ability to discern the effects of different parameters on mRNA expression in the absence of potential confounding reactions caused by toxic drugs. In our version of ATSS, cells were abruptly shifted from $33^{\circ} \mathrm{C}$ to $38^{\circ} \mathrm{C}$ and vice versa, and Cirbp mRNA levels were determined at increasing time intervals after the temperature shift until the new steady state was reached. The principle underlying this strategy is explained in the legend for Figure 3A. Briefly, the time required for reaching $50 \%$ of the new temperature steady-state mRNA levels corresponds to the half-life of the mRNA $\left(T_{1 / 2}\right)$ at that temperature.

The cells were seeded at $\sim 90 \%$ confluence at $37^{\circ} \mathrm{C}$ and incubated for $16 \mathrm{~h}$ at either $33^{\circ} \mathrm{C}$ or $38^{\circ} \mathrm{C}$ in a homemade computer-programmable heating/cooling unit, allowing rapid temperature adjustments. The temperature within the system was then shifted within $10 \mathrm{~min}$ to $38^{\circ} \mathrm{C}$ or $33^{\circ} \mathrm{C}$, respectively, and samples were taken at specific time points after the shift (Fig. 3B). The levels of Cirbp mRNA were subsequently analyzed by multiple methods based on different quantification principles, and the $T_{1 / 2 \mathrm{a}}$ and $T_{1 / 2 \mathrm{~b}}$ values were deduced from the accumulation curves. Figure 3C illustrates the results of Cirbp mRNA (5' untranslated region [UTR]) quantification by qPCR, which yielded average half-lives of $\sim 2 \mathrm{~h}$ for both temperatures. Quantification of Cirbp mRNA by RPA using the intron 6-exon 7 probe gave similar results (Supplemental Fig. S3A-C).

The qPCR analysis showed a relatively high sample variation between data series collected over long time periods with different cell batches. Therefore, we analyzed two sample series-one with high and one with low deviations from the mean trajectory shown in Figure 3C-by Illumina HiSeq RNA-seq to scrutinize the validity of our conclusions. In contrast to qPCR and RPA, which measure only the relative abundances of amplicons and RNA regions complementary to antisense RNA probes, respectively, RNA-seq counts nucleotide segments along all introns and exons of a given transcript. As shown in Figure 4, A and $\mathrm{C}$, the amount of Cirbp exonic reads gradually increased and decreased in cells suddenly shifted to a low or high temperature, respectively. In stark contrast, the amount of intronic reads remained very similar at the two temperatures. In comparison, both the number of exon reads and the number of intron reads in Midn, a gene located $\sim 9 \mathrm{~kb}$ upstream of Cirbp on chromosome 10, remained nearly equal at both temperatures (Fig. 4A,B).

The higher fold change in Cirbp mRNA levels detected by this method enabled us to discern the potential contribution and the extent of effects caused by different post-transcriptional processes that were not possible by the aforementioned approaches. The half-life of Cirbp mRNA in this experiment appeared to be approximately threefold longer at $33^{\circ} \mathrm{C}$ than at $38^{\circ} \mathrm{C}(270 \mathrm{~min}$ vs. 90 min). However, this could not account for the observed $\sim 18$-fold change in the steady-state Cirbp mRNA levels determined by RNA-seq. Thus, we suspected that splicing efficiency, as defined by the fraction of pre-mRNA converted into mature mRNA, must have contributed to temperature-dependent Cirbp mRNA accumulation. To examine this hypothesis, we considered two mathematical models, taking into account different parameters 

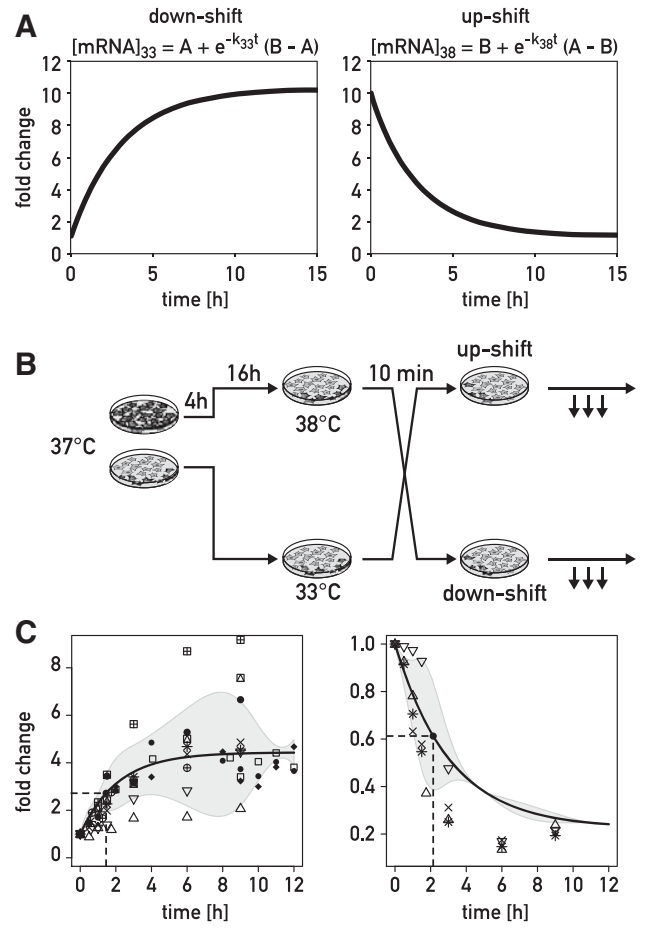

Figure 3. mRNA stability cannot account for temperature-dependent Cirbp mRNA accumulation. (A) Mathematical model for mRNA accumulation of ATSS experiments after a rapid temperature shift, assuming temperature-independent synthesis of mature mRNA (transcription rate and splicing efficiency). The half-life of transcripts $\left(T_{1 / 2}\right)$ at a particular temperature is equal to the time needed to reach half of the new steady-state mRNA levels. $(A, B)$ Steady-state mRNA concentrations at $33^{\circ} \mathrm{C}$ and $38^{\circ} \mathrm{C}$, respectively. $k=\ln 2 / T_{1 / 2}$. (B) Schematic representation of the ATSS experiment. The cells were seeded and kept for $4 \mathrm{~h}$ at $37^{\circ} \mathrm{C}$ before the switch to $33^{\circ} \mathrm{C}$ or $38^{\circ} \mathrm{C}$. After $16 \mathrm{~h}$ of incubation, the temperature was shifted within $10 \mathrm{~min}$ to $38^{\circ} \mathrm{C}$ or $33^{\circ} \mathrm{C}$, respectively, and samples were taken at the indicated time points after the shift. $(C)$ qPCR analysis of Cirbp mRNA expression after temperature downshift (left panel) and upshift (right panel). $n=4$ 11 time course series. Data were normalized to endogenous Ppib levels, which remain constant irrespective of temperature. Symbols represent fold changes between a particular time point and the $t_{0}$ time point of each series. Data were fitted to the equations from $A$. Shaded areas surrounding the fit represent one SD.

that could possibly influence the mRNA output of a gene (Supplemental Fig. S3D,E; Supplemental Material). In the first model (Supplemental Fig. S3D), we assumed that cellular pre-mRNA and mRNA levels are determined by the rates of pre-mRNA synthesis (transcription rate $k_{s}$ ), premRNA degradation $\left(k_{p}\right)$, splicing $(\rho)$, and mRNA degradation $\left(k_{m}\right)$. While this model (named model $1[k p . k s]$ ) was able to fit the data, the obtained parameters were not biologically plausible; in particular, pre-mRNA half-lives were very short, and the splicing efficiency was close to zero (Supplemental Table S1).

We thus hypothesized that an additional mechanism might be at play and introduced a term of pre-mRNA splicing "proneness" $(\alpha)$, which represents the fraction of
pre-mRNAs available for productive splicing into mRNA (Supplemental Fig. S3E; Supplemental Material). This parameter could be influenced by RNA conformation (secondary and ternary structure) and/or RNA-binding proteins. By assessing the probability that a change in any of the selected parameters or a combination of them could result in Cirbp mRNA accumulation at $33^{\circ}$ $\mathrm{C}$ versus $38^{\circ} \mathrm{C}$, we identified the model that described the observed data in the most parsimonious way. This was accomplished by defining constant values for $k_{p}, k_{s}$, and $\rho$ and leaving the $k_{m}$ and $\alpha$ as free variables in the model 2 (kp.ks.rho) (Fig. 4D; Supplemental Tables S1-S3; Supplemental Material). We tested the obtained model (fitted on the RNA-seq data) on our PCR data measuring the expression of Cirbp pre-mRNA (intron 1 amplicon) at a higher time resolution after the temperature shift from different data series. These data were in excellent agreement with the model prediction (Supplemental Fig. S3F).

Our model suggested that the change in Cirbp mRNA half-life could only partially account for the transcript up-regulation at low temperature. Indeed, the splicing efficiency $\left(\alpha \times k_{m} / k_{s}\right)$ of Cirbp pre-mRNA was 5.7 times higher at $33^{\circ} \mathrm{C}$ than at $38^{\circ} \mathrm{C}$. Specifically, the fractions of spliced pre-mRNAs were $48.1 \%$ and $8.5 \%$ at $33^{\circ} \mathrm{C}$ and $38^{\circ} \mathrm{C}$, respectively. Hence, the splicing efficiency appeared to be the strongest determinant of Cirbp mRNA expression (Supplemental Table S3). In agreement with this prediction, the Cirbp-cDNA-LMLucR construct encoding the intron-less Cirbp cDNA showed lower sensitivity to temperature cycles in transient transfections than the full-length gCirbp-LMLucR gene (Fig. 4E). The extent of the oscillations varied somewhat between the cell batches and was more prominent in actively proliferating cells. To determine whether these oscillations in bioluminescence signal were reflecting mRNA levels at different temperatures, we generated stable cell lines using the 3T3 Flp-In system, which enables the site-specific insertion of a single-copy transgene, and quantified the amounts of exogenous transcripts after temperature shifts by qPCR. Our analysis suggested that the oscillations of the Cirbp-cDNA-LMLucR reporter might primarily be a result of the fusion protein characteristics (see above), as the mRNA levels of the transgene appeared similar at $33^{\circ} \mathrm{C}$ and $38^{\circ} \mathrm{C}$ (Supplemental Fig. S3G).

\section{Efficient Cirbp pre-mRNA splicing is required for cold-induced Cirbp mRNA up-regulation}

The requirement of introns for temperature-dependent Cirbp expression in our reporter assays (Fig. 4E) and the mathematical modeling described above suggested that temperature-mediated regulation of splicing efficiency mostly accounted for the cold-inducible Cirbp mRNA accumulation. Therefore, we decided to analyze the process of Cirbp pre-mRNA splicing in more detail.

As Cirbp pre-mRNA levels remained nearly constant at different temperatures, we reasoned that the sum of premRNA splicing and degradation rates (presented as the output of $k_{p}$ and $\alpha$ in this case) also stayed invariable. Low temperatures would favor splicing, whereas high 
Gotic et al.
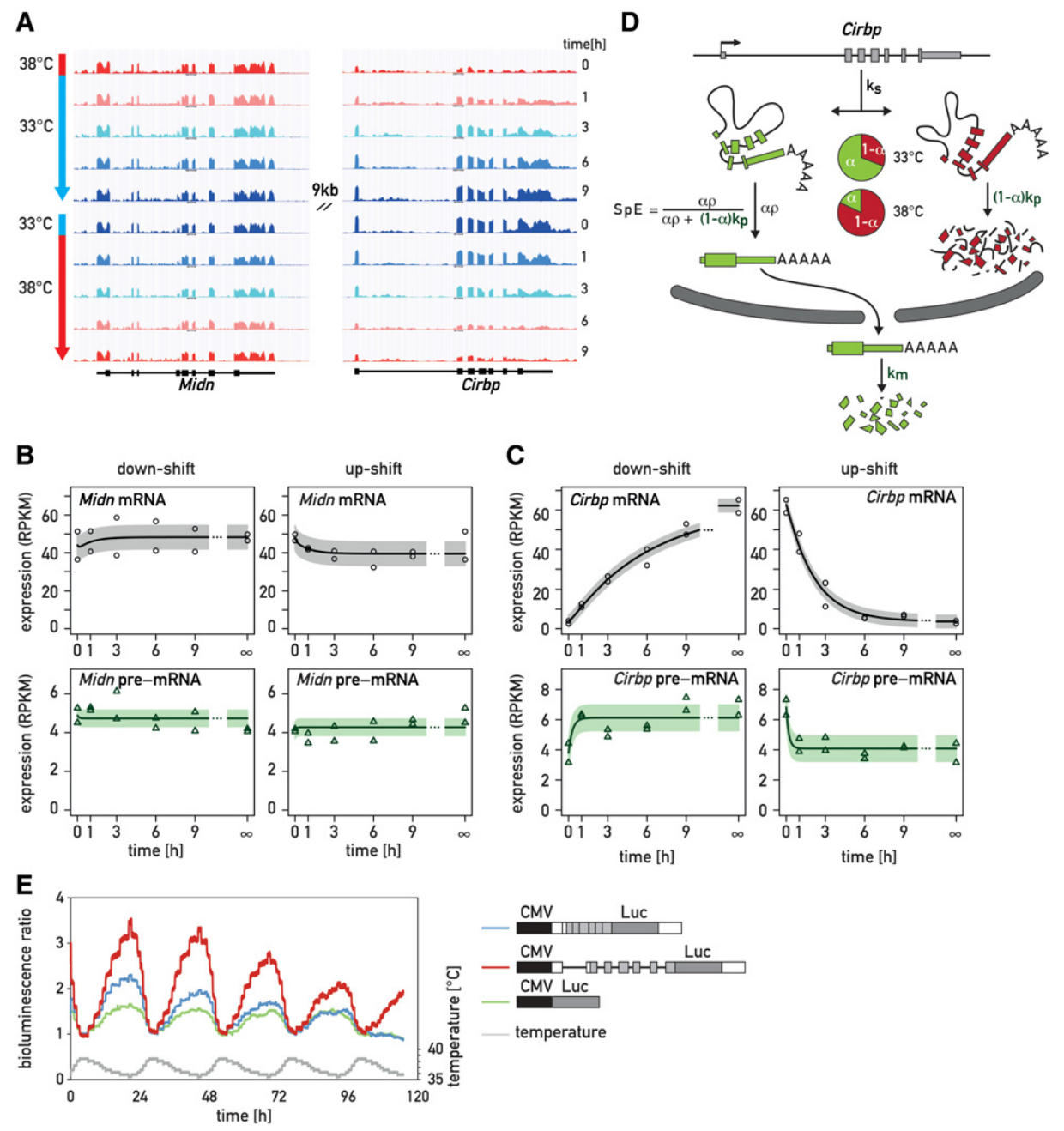

Figure 4. ATSS kinetics based on RNA-seq data. $(A)$ University of California at Santa Cruz genome browser tracks of sequence reads (normalized read density) for Cirbp and Midn, a temperature-unresponsive gene located $9 \mathrm{~kb}$ upstream of Cirbp on chromosome 10. Pooled data from two replicate series (represented by $\Delta$ and $\times$ symbols in downshift and * and $\times$ symbols in upshift experiments in Fig. $3 C$ ) are shown for each temperature shift. Gene structures are depicted below the tracks. $(B, C)$ Quantification of Midn and Cirbp mRNA and pre-mRNA expression for the two biological replicate series/temperature shift. Black circles and green triangles represent the RNA read counts (expressed in RPKM [reads per kilobase per million mapped reads]) for mRNA and pre-mRNA, respectively. Data points indicated by the infinity symbol on the $X$-axis correspond to the expression at $t_{0}$ from the opposite temperature shift and are thus assumed to reflect the opposite steady state. Solid black and green lines represent the model predictions for mRNA and pre-mRNA accumulation. The shaded areas indicate SDs (details are in the Supplemental Material). (D) Schematic representation of the model for temperature-mediated Cirbp regulation. The modeled processes include Cirbp pre-mRNA synthesis $\left(k_{s}\right)$, pre-mRNA degradation $\left(k_{p}\right)$, pre-mRNA splicing $(\rho)$, and mRNA degradation $\left(k_{m}\right)$ rates. In addition, the parameter a reflects the percentage of splicing-prone Cirbp pre-mRNA. The result of the fit indicates similar $k_{s}, k_{p}$, and $\rho$ at $33^{\circ} \mathrm{C}$ and $38^{\circ} \mathrm{C}$, while the $k_{m}$ is threefold lower $(90 \mathrm{vs} .270 \mathrm{~min})$ at $33^{\circ} \mathrm{C}$. Inversely, the splicing efficiency $(\mathrm{SpE})$ appears to be 5.7 times higher $(48 \%$ vs. $8.5 \%)$ at $33^{\circ} \mathrm{C}$. The $\alpha$, represented by the size of the green pie chart slices, increases from $18 \%$ at $38^{\circ} \mathrm{C}$ to $69 \%$ at $33^{\circ} \mathrm{C}$. Together, this generates an 18 -fold change in Cirbp mRNA and a 1.5 -fold change in pre-mRNA levels at $33^{\circ} \mathrm{C}$ versus $38^{\circ} \mathrm{C}$ (Supplemental Table S1-S3). (E) CMV-Cirbp-cDNA-LMLucR reporter assay in transiently transfected cells exposed to simulated CBT cycles. Representative recordings of $n \geq 3$ experiments. The baselines were subtracted in the depicted curves as in Figure 2 . Note that the CMV-gCirbp-LMLucR and CMV-LMLucR reporter tracks in this figure and in Figure 2 look highly similar, manifesting the high reproducibility of the experimental system. Reporter construct schemes are depicted at the right, with Cirbp UTRs presented in white and protein-coding exons in light gray.

temperatures would promote degradation of Cirbp premRNA transcripts while keeping their overall levels almost constant.

To determine the fate of Cirbp pre-mRNA species, we inhibited global splicing by treating the cells with spli- ceostatin A (SSA) and analyzed the levels of the precursors at different temperatures. SSA has been shown to inhibit splicing by binding to the SF3b factor of the U2 splicing complex and to cause an up-regulation of a number of unspliced precursors (Kaida et al. 2007; Koga et al. 2014). 
As expected, adding SSA to NIH3T3 cells prevented the up-regulation of Cirbp mRNA transcripts at $33^{\circ} \mathrm{C}$. However, SSA did not dramatically affect their levels at $38^{\circ} \mathrm{C}$ when compared with the PBS- or methanol-treated samples (Fig. 5A,B; data not shown), indicating a more important role of pre-mRNA splicing at lower temperatures. This was true for samples that were preincubated for 16 $\mathrm{h}$ at different temperatures prior to the SSA treatment and the temperature shift (Fig. 5A) as well as samples preincubated at $37^{\circ} \mathrm{C}$ to ensure equal uptake of the drug by the cells (Fig. 5B). In contrast to the other genes whose pre-mRNAs accumulated during SSA treatment (Kaida et al. 2007; Koga et al. 2014), Cirbp pre-mRNA levels decreased at the same rate at $33^{\circ} \mathrm{C}$ and $38^{\circ} \mathrm{C}$ (Fig. 5C). This could be explained by either increased degradation, as previously shown for a subset of genes in SSA-treated cells (Davidson et al. 2012), or attenuated transcription. However, in cells in which splicing was inhibited by depletion of U2 snRNP, Cirpb expression was reduced by a mechanism other than transcription elongation (data retrieved from Koga et al. 2014).

Since SSA functions as a general splicing inhibitor and since its influence on Cirbp expression might have been a result of secondary effects, we decided to specifically block splicing of Cirbp transcripts by using an antisense phosphorodiamidate morpholino oligomer (AMO) targeting the intron 1-exon 2 boundary of the pre-mRNA. As shown in Figure 5D, AMO treatment increased the levels of intron 1-containing Cirbp precursors at both temperatures compared with control samples, whereas the amount of intron 6-containing Cirbp transcripts re- mained unaltered. This suggested that the AMO acted sequence-specifically on the splicing of the first intron (Fig. $5 \mathrm{D})$. Again, the inhibition of splicing affected the levels of mature Cirbp mRNA only at $33^{\circ} \mathrm{C}$, whereas the mRNA levels at $38^{\circ} \mathrm{C}$ remained unaltered compared with the standard control AMO transfected cells. The temperature-mediated effect was specific for Cirbp, as another AMO targeting the pre-mRNA of Lgals1, a gene whose expression does not significantly change within the range of examined temperatures, did not show such a pattern. Importantly, AMO treatment caused a higher increase of Cirbp pre-mRNA at $33^{\circ} \mathrm{C}$ than at $38^{\circ} \mathrm{C}$, whereas the Lgals1 pre-mRNA targeting AMO provoked similar rises of Lgals1 pre-mRNA at both temperatures (Fig. 5D). An additional Cirbp AMO targeting the intron 4-exon 5 boundary showed a similar effect on Cirpb pre-mRNA transcript levels (Supplemental Fig. S4A). The total amount of Cirbp mRNA in this case did not change due to alternative splicing caused by the AMO (Supplemental Fig. S4A). These data supported the conclusion based on the above-described ATSS experiments that Cirbp pre-mRNA splicing is more efficient at reduced temperatures.

Given that transcription rates and pre-mRNA steadystate concentrations were nearly temperature-insensitive, the sum of splicing-prone and degradation-prone Cirbp pre-mRNAs must have been similar at the examined temperatures. What appeared to change at $33^{\circ} \mathrm{C}$ and $38^{\circ} \mathrm{C}$ were the fractions of splicing-prone and degradation-prone pre-mRNAs. The predicted noncanonical splicing sites and functional RNA elements within the first Cirbp
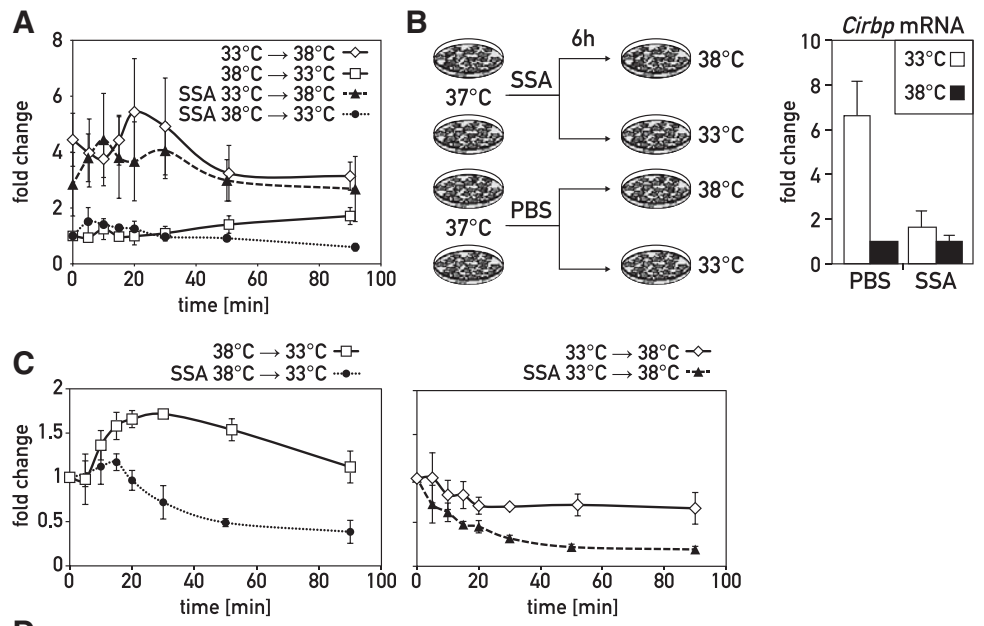

D

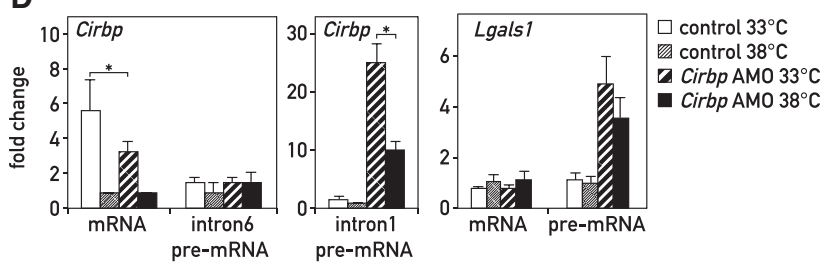

Figure 5. Efficient Cirbp pre-mRNA splicing is required for cold-induced Cirbp mRNA up-regulation. $(A)$ qPCR analysis of Cirbp mRNA expression in control and SSA-treated samples after temperature upshifts and downshifts. After an incubation period of $16 \mathrm{~h}$ at $33^{\circ} \mathrm{C}$ or $38^{\circ} \mathrm{C}$, the drug was added, and the cells were shifted to the opposite temperatures for increasing time intervals. $(B$, left panel) Schematic representation of the experiment. Cells were incubated at $37^{\circ} \mathrm{C}$, and SSA or PBS was added immediately before the temperature shift to $33^{\circ} \mathrm{C}$ or $38^{\circ} \mathrm{C}$. Samples were collected after $6 \mathrm{~h}$ of incubation and analyzed by qPCR. (Right panel) qPCR analysis of Cirbp expression after the addition of $20 \mathrm{ng} / \mathrm{mL}$ SSA. $n=3$ independent experiments. Data were normalized to endogenous Ppib levels and are presented as fold changes between a particular sample and the $38^{\circ} \mathrm{C}$ PBS sample of a series. (C) qPCR analysis of Cirbp premRNA expression in control and SSA-treated samples after temperature downshift (left panels) and upshift (right panels). (D) qPCR analysis of Cirbp and Lgals1 transcripts in antisense phosphorodiamidate morpholino oligomer (AMO)-treated (Cirbp/Lgals1 AMO mix) and control (standard control oligo) samples $16 \mathrm{~h}$ after transfection/temperature shift. $n=2$ series of three biological replicates. Data were normalized to endogenous Ppib levels and are presented as fold changes between a particular sample and a control $38^{\circ} \mathrm{C}$ sample of the same series. $\left(^{*}\right) P<0.05, t$-test assuming unequal variances. $(A, C) n=3$ independent series. Data were normalized to endogenous $P$ pib levels and are presented as fold changes between a particular time point and the 0 time point of each series. The bars represent SDs. 
intron (Supplemental Table S4) could have played a role in this process.

Since intronic sequences have been implicated in the mechanisms targeting pre-mRNA for degradation (Kilchert et al. 2015), we attempted to identify the potential degradation pathways involved in the temperature-mediated Cirbp pre-mRNA regulation by knocking down putative factors of the cytoplasmic (Xrn1) and nuclear (Xrn2 and Dom3z) 5' $\rightarrow 3^{\prime}$ quality control mechanisms. However, the down-regulation of these factors did not have any major effects on Cirbp mRNA expression (Supplemental Fig. S4B-F). In contrast, the knockdown of Exosc9 and Exosc10, representing the core and nuclear catalytic components of the exosome, a $3^{\prime} \rightarrow 5^{\prime}$ RNA degradation complex (for review, see Eberle and Visa 2014), showed a moderate up-regulation of Cirbp pre-mRNA accumulation irrespective of temperature (Supplemental Fig. S4D,E)

\section{A splicing-dependent regulatory element in Cirbp intron 1 mediates temperature-responsive Cirbp expression}

Since the luciferase reporter construct bearing the fulllength Cirbp gene showed temperature-responsive expression (Fig. 2C), we set out to narrow down the putative temperature-sensing element. To this end, we generated a series of deletion constructs fused to the luciferase gene and tested their ability to respond to simulated CBT cycles. As shown in Figure 6, 337 bp of the truncated Cirbp intron $1(\Delta 315-1870)$ were sufficient to confer temperature sensitivity to the luciferase reporter, whether situated in the native Cirbp exon 1-exon $25^{\prime}$ UTR-encoding position (Fig. 6A,B, construct 4), between different exons of the same gene, or between exons of the temperature-irresponsive control gene Coasy (Fig. 6C, construct 6). Placing the Cirbp intron sequence after the luciferase gene did not induce the same effect, suggesting the requirement of splicing in the temperature-responsive process. This characteristic was specific for Cirbp intron 1, as other Cirbp introns (Fig. 6C, constructs 10-14) and the Coasy intron 1 (Fig. 6C, construct 15) did not confer temperature sensitivity to reporter genes (Fig. 6A,D). The most important region within the $337 \mathrm{bp}$ of the truncated Cirbp intron 1 probably lies at the $3^{\prime}$ end, comprising the predicted branch point sites, as another Cirbp construct with a deletion in the $5^{\prime}$ region of intron 1 still showed some temperature responsiveness (Supplemental Fig. S5A, construct B).

Although Cirbp intron 1 was sufficient to confer temperature sensitivity to a reporter gene, full-length constructs without the Cirbp intron 1 showed temperature oscillations depending on the proliferation and the transformation state of the NIH3T3 cells (Supplemental Fig. S5A, constructs C and D)-similar to the aforementioned Cirbp-cDNA-LMLucR construct-and are likely a consequence of the fusion protein characteristics. Interestingly, placing the Cirbp gene at the $3^{\prime}$ terminus of the luciferase reporter abolishes temperature sensitivity of the construct (Supplemental Fig. S5B, construct E). This might be due to a changed structure of the transcript or a different splicing enhancer/suppressor environment that favors the use of another splice donor site within the luciferase
A

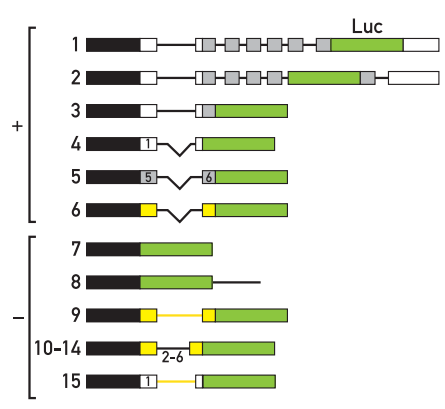

B

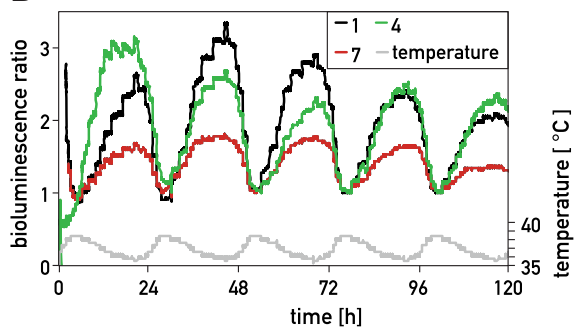

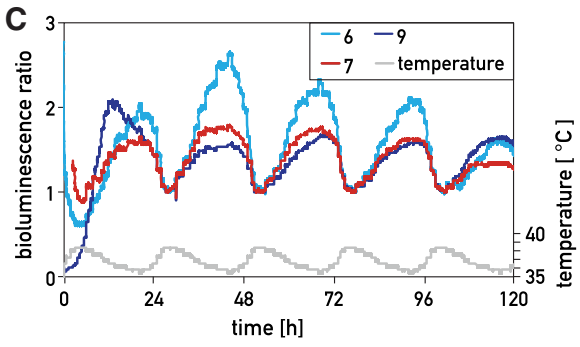

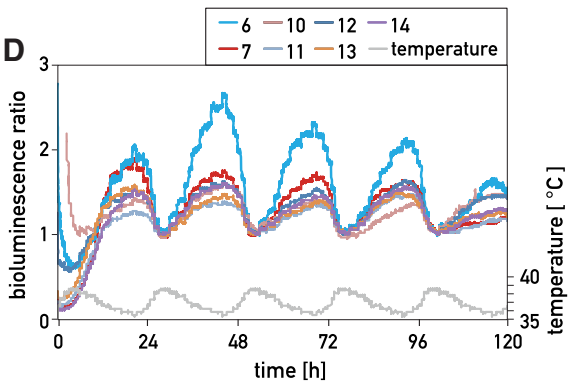

Figure 6. A cis-acting element in Cirbp intron 1 contributes to temperature-mediated Cirbp expression. $(A)$ Schematic representation of Cirbp-luciferase reporter constructs with $(+)$ or without $(-)$ temperature-dependent expression. White bars illustrate UTR regions. Bioluminescence tracks of gCirbp-LMLucR (1) and Cirbp5'UTR-Int1DelB-LMLucR (4) constructs (B), Coasy5'UTR-LMLucR (9) and Coasy5'UTR-CirbpInt1DelB-LMLucR (6) constructs $(C)$, and Coasy5'UTR-Cirbp(Int1DelB-Int6)-LMLucR (6, 10-14) constructs (D). The recordings of the control LMLucR reporter shown in $B$ and $C$ and the tracks of construct 6 in $C$ and $D$ were part of the same experiment. $n \geq 3$ replicates for each track. Data were normalized to baseline curves. 
gene to be spliced with the Cirbp exon 2 acceptor site in a fraction of transcripts (Supplemental Fig. S5C).

\section{Genome-wide analysis reveals a predominant role of temperature in regulating pre-mRNA splicing efficiency and $m R N A$ stability}

We wished to examine whether the regulatory mechanisms described above were specific for Cirbp or played a more general role in the control of temperature-dependent gene expression. To this end, we performed a genome-wide analysis of our ATSS RNA-seq data set described in Figure 4. Out of 24,756 detected genes, we identified 201 thermoregulated genes-63 induced by cold and 138 induced by heat (Fig. 7A; Supplemental Fig. S6A; Supplemental Table S5; Supplemental Material). Gene ontology analysis revealed that these genes belong to different functional categories, as shown in Supplemental Table S6. If these genes were temperature-regulated in vivo, their transcripts would be expected to accumulate diurnally in mouse livers. Figure $7 \mathrm{~B}$ shows that the mRNA accumulation of a significant number of cold-inducible and heat-inducible genes $\left(P=1.9 \times 10^{-7}\right.$ and $P=$ 0.014 , respectively, hypergeometric test) exhibited a robust oscillation $(P<0.05$, harmonic regression, $F$-test $)$, with peak values occurring during the day (low CBT) for the cold-inducible genes and during the night (high CBT) for the heat-inducible genes.

We applied the mathematical model described in Figure $4 \mathrm{D}$ to determine the most probable temperature-dependent regulation mechanisms for those cold-inducible and heat-inducible genes whose transcripts reached a new steady-state concentration after the temperature shift (32 cold-inducible and 99 heat-inducible genes) (Fig. 7C; Supplemental Figure S6B; Supplemental Table S5). The genes whose pre-mRNA levels were transiently induced (Supplemental Fig. S6C; Supplemental Table S5) were excluded from the analysis, as the underpinning mechanisms could not be deduced from the ATSS model. Taking into account the mRNA and pre-mRNA expression patterns of the genes reaching a new steady-state concentration, we were able to estimate the probability of the temperature influence on a particular RNA synthesis or processing parameter for each gene (Supplemental Material). As shown in Figure 7D, $\alpha$ (defining the splicing "proneness" of the pre-mRNA population) and $k_{m}$ (determining the stability of mature mRNAs) were the parameters most frequently affected by temperature for these groups of genes. Hence, for a subclass of temperature-dependent genes, post-transcriptional regulatory mechanisms appeared to play a prevalent role.

Since Cirbp was the cold-inducible gene with the highest change in expression in our ATSS analysis, we asked whether its presence was required for the general temperature-mediated response in cells. To address this question, we examined whether the heat-inducible and cold-inducible candidates were enriched with previously published CIRBP-bound RNAs (Morf et al. 2012). As shown in Supplemental Table S7, the cold-inducible genes were enriched with CIRBP targets, especially those bound by
CIRBP in their $3^{\prime}$ UTRs $(P=0.0014$, hypergeometric test). For cold-inducible CIRBP-bound transcripts, $k_{m}$ was the most frequently affected parameter regulating temperature-dependent mRNA accumulation, whereas for the heat-inducible CIRBP-bound transcripts, a and $k_{m}$ played similarly important roles in this process (Supplemental Table S5).

Surprisingly, $21.7 \%$ of pre-mRNAs in the heat-inducible group were found to be bound by CIRBP, in contrast to only $9.5 \%$ of the cold-inducible pre-mRNAs $\left(P=1.105 \times 10^{-7}\right.$ and $P=0.2409$, respectively, hypergeometric test). In accordance with these observations, the knockdown of Cirbp at $33^{\circ} \mathrm{C}$ (Morf et al. 2012) resulted in a significant decrease in heat-inducible gene expression (Fig. 7E). However, the transcript levels of cold-inducible CIRBP targets were not affected by its absence. Hence, the role of CIRBP, if any, for cold-inducible mRNAs is not related to their accumulation at low temperature but perhaps to their subcellular distribution or translatability. Taken together, these data imply a protective/regulatory role of CIRBP for heat-inducible transcripts at lower temperatures.

\section{Discussion}

The effect of temperature on mammalian gene expression has thus far been studied mainly in the context of socalled cold-shock and heat-shock responses, which are reactions to conditions posing a threat for cellular function and integrity (for review, see Fujita 1999; Miozzo et al. 2015). In this study, we determined gene-specific and genome-wide changes in transcript expression caused by temperature values within the physiological range $\left(33^{\circ} \mathrm{C}\right.$ $<\mathrm{T}<39^{\circ} \mathrm{C}$ ). Our studies revealed a novel temperature-dependent post-transcriptional regulation mechanism governing the expression of CIRBP, a cold-inducible RNAbinding protein involved in the modulation of circadian gene expression. In addition, our data suggested the existence of general body temperature-mediated gene regulation pathways that depend in part on the activity of the CIRBP protein.

Temperature-controlled alternative splicing has been implicated previously in the regulation of gene expression in various organisms (Cizdziel et al. 1988; Diernfellner et al. 2007; Cao and Edery 2015). However, to the best of our knowledge, the impact of temperature on splicing efficiency, as defined in this study, has not yet been reported. Here, we present a novel, temperature-dependent posttranscriptional mechanism that modulates gene output by determining the fraction of pre-mRNA processed into mature functional mRNA. In contrast to the well-studied process of alternative splicing, the mechanism that we describe governs temperature-dependent accumulation of one and the same mRNA species.

The "splice or die" fate of the Cirbp precursors might be determined by temperature-sensitive RNA secondary structures influencing splice site recognition and spliceosome binding, as described for some viruses (Abbink and Berkhout 2008). This hypothesis is supported by the predicted existence of noncanonical donor and acceptor splice 
A

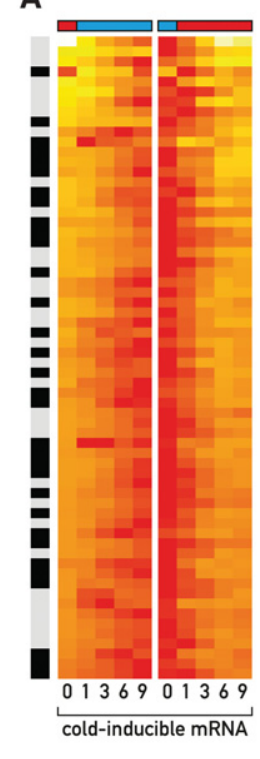

B

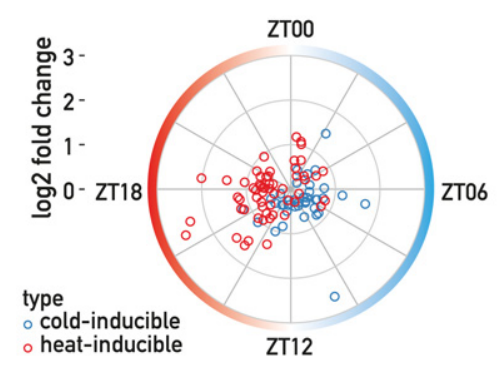

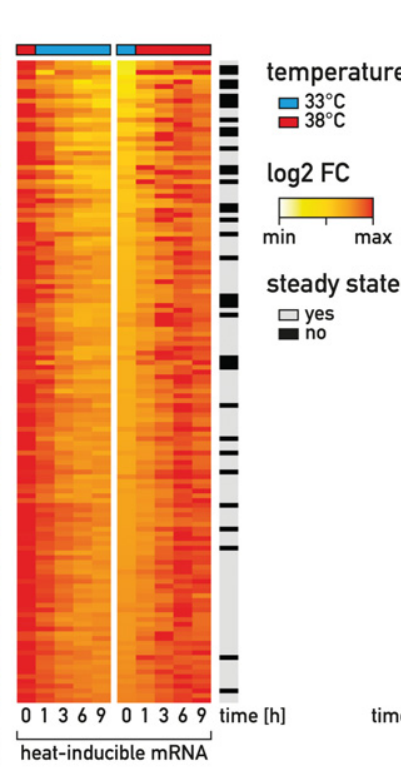

D

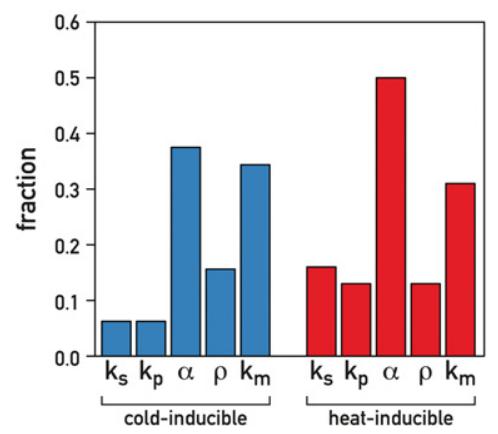

C

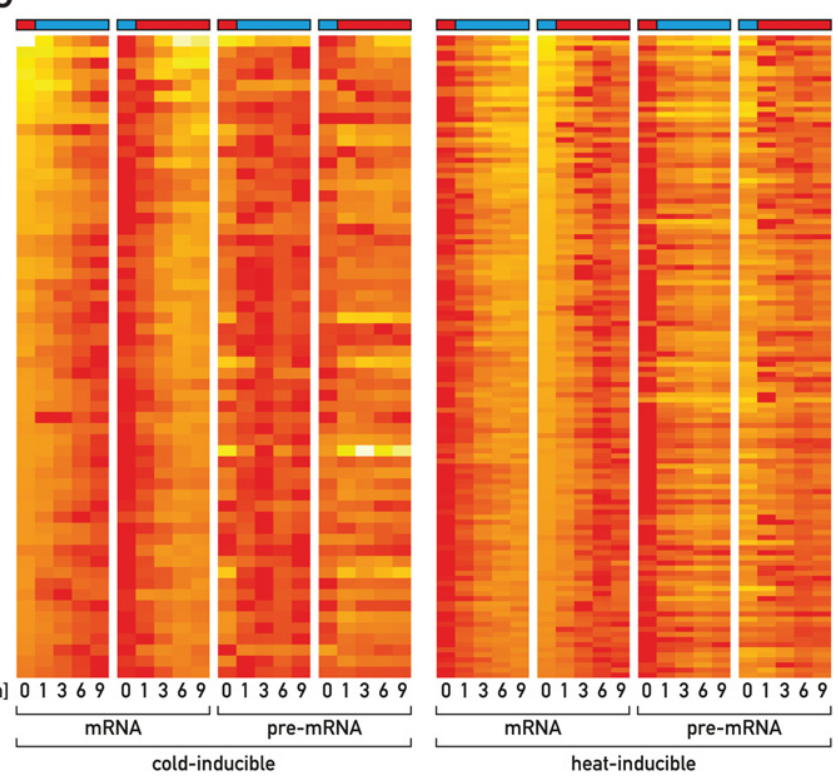

E

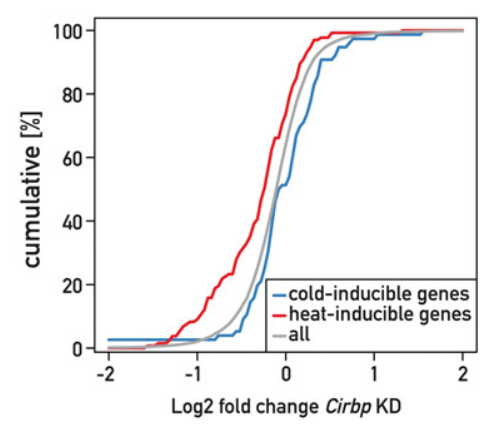

Figure 7. Genome-wide transcript analysis reveals a predominant role of splicing efficiency and mRNA stability in the temperaturedependent accumulation of mRNAs. (A) Heat maps of 63 cold-inducible (left panel) and 138 heat-inducible (right panel) genes detected in ATSS RNA-seq analysis after temperature downshifts and upshifts. Color indicates $\log _{2}$ fold changes (FC) with respect to the maximum mRNA expression for each gene over all time points. Genes are ordered according to their maximum fold changes. Black bars mark genes with transient temperature responses, as opposed to genes whose transcripts reach a new steady state after the temperature shift (gray) (details are in the Supplemental Material). (B) Polar plot showing phases and fold changes of expression (calculated by the peak to trough) for cold-inducible (blue circles) and heat-inducible (red circles) genes that exhibited circadian expression patterns in mouse livers. $P<0.05$, harmonic regression; data retrieved from Atger et al. (2015). The red-blue gradient circle represents mouse CBT values around the clock, with red and blue symbolizing high and low temperatures, respectively. $(C)$ Temporal abundance profiles for mRNAs and pre-mRNAs for 32 cold-inducible and 99 heat-inducible intron-containing genes from $A$ that reached a new steady state. Fold changes for mRNAs and pre-mRNAs were calculated with respect to the maximum expression levels of mRNAs and pre-mRNAs. $(D)$ The charts depict the fraction of genes in the cold-inducible (blue) and heat-inducible (red) groups showing evidence of temperature dependence for any of the parameters (probability $>0.5$ ) (Supplemental Material). (E) Cumulative distribution of mRNA accumulation in cells in which Cirbp expression was depleted by siRNA knockdown (KD). The $X$-axis indicates $\log _{2}$ fold changes between Cirbp knockdown and wild type (Morf et al. 2012). The gray line corresponds to all genes. Cirbp depletion affects cold-inducible (blue) and heat-inducible (red) genes differently. $P=0.0034$, Mann-Whitney-Wilcoxon test. Upon Cirbp knockdown, heat-inducible genes show a reduction in their mRNA accumulation, whereas cold-inducible genes remain unaffected. $P=4.5 \times 10^{-7}$ and $P=0.78$, respectively, $t$-test.

sites and functional RNA structures in Cirbp intron 1 as well as the ability of the same intron to convey temperature sensitivity to an otherwise CBT-irresponsive gene.

Increased levels of Cirbp mRNA and protein upon mild cold exposure have been attributed previously to transcriptional control mechanisms (Sumitomo et al. 2012). However, our present study—showing similar RNA poly- merase II occupancy at the Cirbp gene and comparable pre-mRNA levels in NIH3T3 cells at different temperatures-excludes the control of primary transcript synthesis as the major regulatory mechanism. In keeping with this conclusion, a recent study analyzing genome-wide RNA polymerase II occupancy in mouse livers collected at different time points around the clock revealed a 
similar density of RNA polymerase II molecules on Cirbp throughout the day (Le Martelot et al. 2012). However, Cirbp mRNA and protein accumulation follows a robust diurnal cycle whose phase is nearly anti-phasic to that of the CBT oscillation. Moreover, replacing the Cirbp promoter with the CMV promoter did not abolish temperature-sensitive Cirbp expression. However, there remains the possibility of a structure/splicing-affecting factor being handed off to an intronic sequence during transcription, which could subsequently affect the downstream processing of the transcript.

Cis-acting Cirbp promoter elements binding the transcription factor Sp1 have been proposed previously to participate in cold-inducible Cirbp transcription (Sumitomo et al. 2012). However, in our hands, the depletion of endogenous Sp1 by RNAi to $<35 \%$ of its original concentration had no effect on the temperature-dependent Cirbp mRNA accumulation (data not shown).

Different temperature-specific Cirbp mRNA isoforms arising through the use of alternative TSSs have been reported in a previous study (Al-Fageeh and Smales 2009). By using a variety of methods including 5 '-RACE, Northern blot experiments, and deep sequencing of RNA harvested from cells incubated at different temperatures, we detected only one major Cirbp mRNA species initiating at the sequence ACTCGCGCCTTAGG irrespective of the incubation temperature. This transcript corresponds to the one initially reported by Nishiyama et al. (1997).

Post-transcriptional regulatory mechanisms can act on both the accumulation and function of mRNAs. Often, they involve cis-acting RNA elements that modulate translation efficiency or transcript stability (for review, see Adjibade and Mazroui 2014). The diminished temperature sensitivity of the Cirbp cDNA reporter construct, which encompasses the entire sequence of mature Cirbp mRNA, supports our conclusion that temperature-dependent mRNA instability was unlikely to be the sole parameter accounting for cold-inducible Cirbp expression. The 3' UTR of Cirbp mRNA does contain a predicted AUrich element known to destabilize many mRNAs. Indeed, the half-life of Cirbp RNA is rather short, and the AU-rich element may contribute to the destabilization of this transcript irrespective of temperature. In accordance, a general increase of Cirbp mRNA in Dicer-deficient mice rules out a temperature-specific miRNA-dependent regulatory mechanism (data provided by Du et al. 2014). The treatment of cells with the general splicing inhibitor SSA provided additional support against a major effect of temperature on Cirbp mRNA stability. Namely, the levels of Cirbp mRNA were similar at both temperatures even after a 6-h treatment of the cells with SSA. If the half-life of Cirbp mRNA was markedly longer at $33^{\circ} \mathrm{C}$, the level of transcripts would have been expected to decrease more slowly at $33^{\circ} \mathrm{C}$ than at $38^{\circ} \mathrm{C}$. The absence of detectable effects on Cirbp mRNA levels at $38^{\circ} \mathrm{C}$ in SSA-treated cells might be due to the intrinsically low level of splicing at that temperature (in accordance with splicing efficiency of $8.5 \%$ ), some of which still persisted at the SSA concentration that we used.
This and other studies underscore the large impact that relatively mild temperature changes can exert on different aspects of splicing. As mentioned above, various reports have demonstrated that temperature can affect splice site selection, resulting in the modulation of alternative splicing. Furthermore, a recent study showed that Sfrs 10 (SRp38), a splicing repressor, attenuates the splicing of a large number of transcripts in human cells exposed to heat shock (Shalgi et al. 2014). By recording ATSS kinetics for both mature mRNAs and pre-mRNAs, we could identify splicing efficiency as an important parameter in the temperature-dependent regulation of Cirbp mRNA. Finally, the simple mathematical modeling that we applied to the transcriptome-wide ATSS analysis revealed that moderate differences in temperature may have a large effect on the splicing efficiency and stability of many temperaturesensitive transcripts.

\section{Materials and methods}

Cell culture, siRNA, and morpholino transfections

NIH3T3 cells were cultured under standard conditions. Stable cell lines expressing Cirbp reporter constructs were generated using the 3T3 Flp-In/pCDNA5/FRT system (Invitrogen) according to the manufacturer's instructions. For the SSA treatment, the medium was supplemented with $20 \mathrm{ng} / \mathrm{mL}$ SSA (a generous gift from Minoru Yoshida, RIKEN, Institute of Physical and Chemical Research, Saitama, Japan), and the cells were incubated for a maximum of $6 \mathrm{~h}$.

The AMOs targeting the Cirbp intron 1-exon 2 border (19561972), the Cirbp intron 4-exon 5 border (2565-2592), and the Lgals intron 1-exon 2 border (1225-1250) were purchased from Gene Tools together with the standard control oligo. The constructs were delivered into cells by electroporation using Amaxa Cell Line Nucleofector kit R and the Nucleofector device (Lonza) as recommended by the manufacturer. Cells were transfected with $50 \mu \mathrm{M}$ oligo mix ( $25 \mu \mathrm{M}$ Cirbp $+25 \mu \mathrm{M}$ Lgals1) or standard control oligo per $3 \times 10^{6}$ cells and were switched directly to different temperatures after transfection for $18 \mathrm{~h}$.

Transient gene-specific knockdown experiments were done by transfection of ON-TARGETplus SMART pool siRNAs (Dharmacon) using Lipofectamine RNAiMAX from Invitrogen according to manufacturer's instructions. siRNAs targeting mRNAs specified by the following mouse genes were used: Xrn1, Xrn2, Dom3z, Exosc9, Exosc10, Fus, and Nono. The cells were allowed to settle for $4 \mathrm{~h}$ at $37^{\circ} \mathrm{C}$ after transfection and then were shifted for $72 \mathrm{~h}$ to different temperatures before RNA isolation.

RBPDB (the database of RNA-binding protein specificities, version 1.3 release; http://rbpdb.ccbr.utoronto.ca) was used for scanning the Cirbp sequence for potential RBP-binding sites. The search for splice sites and cis-acting elements was performed on the RegRNA 2.0 (Chang et al. 2013) and the ASSP (Wang and Marin 2006) Web servers. Gene ontology analysis was performed using DAVID Bioinformatics resources 6.8 analysis Web server (https://david-d.ncifcrf.gov).

RNA isolation and quantification, ChIP, and bioluminescence reporter assays were performed according to standard methods described in detail in the Supplemental Material.

RNA-seq and data analysis

DNase I-treated total RNA samples were ribo-depleted using the Ribo-Zero Gold rRNA removal kit (Illumina). Paired-end, strand- 
specific cDNA libraries of 50-bp inserts were prepared according to an in-house protocol of the iGE3 genomics platform and sequenced using the Illumina HiSeq2500 platform. Detailed information on data analysis and mathematical modeling is in the Supplemental Material. Data have been deposited to the Gene Expression Omnibus repository under accession number GSE85553.

\section{Acknowledgments}

We thank Minoru Yoshida (RIKEN, Institute of Physical and Chemical Research, Saitama, Japan) for the generous gift of SSA, and M. Koksharov and N. Ugarova (University of Moscow, Russia) for the Luciola mingrelica luciferase mutant. We are extremely grateful to André Liani, Yves-Alain Poget, and Georges Severi (Mechanical Workshop Unit of the Department of Molecular Biology, University of Geneva) for designing and engineering the homemade incubators with electronic temperature controls. We also express our gratitude to Mylène Docquier and her collaborators (Genomics Platform, University of Geneva) for RNA-seq, and Nicolas Roggli (Department of Molecular Biology, University of Geneva) for the artwork. The computations were performed on the Vital-IT (http://www.vital-it.ch) Center for High-Performance Computing of the Swiss Institute of Bioinformatics (SIB). Work in the laboratory of U.S. was supported by the Swiss National Science Foundation (SNF 31-113565 and SNF 31-128656/1), the European Research Council (ERC-AdG TimeSignal), the State of Geneva, and the Louis Jeantet Foundation of Medicine. Work in the laboratory of F.N. was supported by the Swiss National Science Foundation (SNF 31-130714) and the Ecole Polytechnique Fédérale de Lausanne (EPFL). I.G. received a long-term Federation of European Biochemical Societies (FEBS) fellowship (2010-2012).

\section{References}

Abbink TE, Berkhout B. 2008. RNA structure modulates splicing efficiency at the human immunodeficiency virus type 1 major splice donor. J Virol 82: 3090-3098.

Adjibade P, Mazroui R. 2014. Control of mRNA turnover: implication of cytoplasmic RNA granules. Semin Cell Dev Biol 34: 15-23.

Al-Fageeh MB, Smales CM. 2009. Cold-inducible RNA binding protein (CIRP) expression is modulated by alternative mRNAs. RNA 15: 1164-1176.

Atger F, Gobet C, Marquis J, Martin E, Wang J, Weger B, Lefebvre G, Descombes P, Naef F, Gachon F. 2015. Circadian and feeding rhythms differentially affect rhythmic mRNA transcription and translation in mouse liver. Proc Natl Acad Sci 112: E6579-E6588.

Bensaude O. 2011. Inhibiting eukaryotic transcription: which compound to choose? How to evaluate its activity? Transcription 2: 103-108.

Brown SA, Zumbrunn G, Fleury-Olela F, Preitner N, Schibler U. 2002. Rhythms of mammalian body temperature can sustain peripheral circadian clocks. Curr Biol 12: 1574-1583.

Buhr ED, Yoo SH, Takahashi JS. 2010. Temperature as a universal resetting cue for mammalian circadian oscillators. Science 330: 379-385.

Cao W, Edery I. 2015. A novel pathway for sensory-mediated arousal involves splicing of an intron in the period clock gene. Sleep 38: 41-51.

Chang TH, Huang HY, Hsu JB, Weng SL, Horng JT, Huang HD. 2013. An enhanced computational platform for investigating the roles of regulatory RNA and for identifying functional RNA motifs. BMC Bioinformatics 14: S4.
Cizdziel PE, de Mars M, Murphy EC Jr. 1988. Exploitation of a thermosensitive splicing event to study pre-mRNA splicing in vivo. Mol Cell Biol 8: 1558-1569.

Davidson L, Kerr A, West S. 2012. Co-transcriptional degradation of aberrant pre-mRNA by Xrn2. EMBO J 31: 2566-2578.

Dibner C, Schibler U, Albrecht U. 2010. The mammalian circadian timing system: organization and coordination of central and peripheral clocks. Annu Rev Physiol 72: 517-549.

Diernfellner A, Colot HV, Dintsis O, Loros JJ, Dunlap JC, Brunner M. 2007. Long and short isoforms of Neurospora clock protein FRQ support temperature-compensated circadian rhythms. FEBS Lett 581: 5759-5764.

Du NH, Arpat AB, De Matos M, Gatfield D. 2014. MicroRNAs shape circadian hepatic gene expression on a transcriptomewide scale. Elife 3: $\mathrm{e} 02510$.

Eberle AB, Visa N. 2014. Quality control of mRNP biogenesis: networking at the transcription site. Semin Cell Dev Biol 32: $37-46$.

Fornace AJ Jr, Alamo I Jr, Hollander MC. 1988. DNA damage-inducible transcripts in mammalian cells. Proc Natl Acad Sci 85: 8800-8804.

Fraschini A, Bottone MG, Scovassi AI, Denegri M, Risueno MC, Testillano PS, Martin TE, Biggiogera M, Pellicciari C. 2005. Changes in extranucleolar transcription during actinomycin D-induced apoptosis. Histol Histopathol 20: 107-117.

Fujita J. 1999. Cold shock response in mammalian cells. J Mol Microbiol Biotechnol 1: 243-255.

Greenberg JR. 1972. High stability of messenger RNA in growing cultured cells. Nature 240: 102-104.

Hamid AA, Mandai M, Fujita J, Nanbu K, Kariya M, Kusakari T, Fukuhara K, Fujii S. 2003. Expression of cold-inducible RNAbinding protein in the normal endometrium, endometrial hyperplasia, and endometrial carcinoma. Int I Gynecol Pathol 22: $240-247$.

Harrold S, Genovese C, Kobrin B, Morrison SL, Milcarek C. 1991. A comparison of apparent mRNA half-life using kinetic labeling techniques vs decay following administration of transcriptional inhibitors. Anal Biochem 198: 19-29.

Kaida D, Motoyoshi H, Tashiro E, Nojima T, Hagiwara M, Ishigami K, Watanabe H, Kitahara T, Yoshida T, Nakajima H, et al. 2007. Spliceostatin A targets SF3b and inhibits both splicing and nuclear retention of pre-mRNA. Nat Chem Biol 3: 576-583.

Kilchert C, Wittmann S, Passoni M, Shah S, Granneman S, Vasiljeva L. 2015. Regulation of mRNA levels by decay-promoting introns that recruit the exosome specificity factor Mmi1. Cell Rep 13: 2504-2515.

Koga M, Satoh T, Takasaki I, Kawamura Y, Yoshida M, Kaida D. 2014. U2 snRNP is required for expression of the $3^{\prime}$ end of genes. PLoS One 9: e98015.

Koksharov MI, Ugarova NN. 2011. Thermostabilization of firefly luciferase by in vivo directed evolution. Protein Eng Des Sel 24: 835-844.

Koksharov MI, Ugarova NN. 2012. Approaches to engineer stability of beetle luciferases. Comput Struct Biotechnol I 2: e201209004.

Kornmann B, Schaad O, Bujard H, Takahashi JS, Schibler U. 2007. System-driven and oscillator-dependent circadian transcription in mice with a conditionally active liver clock. PLoS Biol 5: e34.

Le Martelot G, Canella D, Symul L, Migliavacca E, Gilardi F, Liechti R, Martin O, Harshman K, Delorenzi M, Desvergne $\mathrm{B}$, et al. 2012. Genome-wide RNA polymerase II profiles and RNA accumulation reveal kinetics of transcription and associated epigenetic changes during diurnal cycles. PLOS Biol 10: e1001442. 
Liu Y, Hu W, Murakawa Y, Yin J, Wang G, Landthaler M, Yan J. 2013. Cold-induced RNA-binding proteins regulate circadian gene expression by controlling alternative polyadenylation. Sci Rep 3: 2054.

Masuda T, Itoh K, Higashitsuji H, Nakazawa N, Sakurai T, Liu Y, Tokuchi H, Fujita T, Zhao Y, Nishiyama H, et al. 2012. Coldinducible RNA-binding protein (Cirp) interacts with Dyrk1b/ Mirk and promotes proliferation of immature male germ cells in mice. Proc Natl Acad Sci 109: 10885-10890.

Miozzo F, Saberan-Djoneidi D, Mezger V. 2015. HSFs, stress sensors and sculptors of transcription compartments and epigenetic landscapes. J Mol Biol 427: 3793-3816.

Morf J, Rey G, Schneider K, Stratmann M, Fujita J, Naef F, Schibler U. 2012. Cold-inducible RNA-binding protein modulates circadian gene expression posttranscriptionally. Science 338: 379-383.

Nishiyama H, Itoh K, Kaneko Y, Kishishita M, Yoshida O, Fujita J. 1997. A glycine-rich RNA-binding protein mediating cold-inducible suppression of mammalian cell growth. I Cell Biol 137: 899-908.

Nishiyama H, Xue JH, Sato T, Fukuyama H, Mizuno N, Houtani T, Sugimoto T, Fujita J. 1998. Diurnal change of the cold-inducible RNA-binding protein (Cirp) expression in mouse brain. Biochem Biophys Res Commun 245: 534-538.

Refinetti R. 2010. The circadian rhythm of body temperature. Front Biosci 15: 564-594.

Refinetti R, Kaufman CM, Menaker M. 1994. Complete suprachiasmatic lesions eliminate circadian rhythmicity of body temperature and locomotor activity in golden hamsters. I Comp Physiol A 175: 223-232.

Reinke H, Saini C, Fleury-Olela F, Dibner C, Benjamin IJ, Schibler U. 2008. Differential display of DNA-binding proteins reveals heat-shock factor 1 as a circadian transcription factor. Genes Dev 22: 331-345.

Ren WH, Zhang LM, Liu HQ, Gao L, Chen C, Qiang C, Wang XL, Liu CY, Li SM, Huang C, et al. 2014. Protein overexpression of CIRP and TLR4 in oral squamous cell carcinoma: an immunohistochemical and clinical correlation analysis. Med Oncol 31: 120 .
Saini C, Morf J, Stratmann M, Gos P, Schibler U. 2012. Simulated body temperature rhythms reveal the phase-shifting behavior and plasticity of mammalian circadian oscillators. Genes Dev 26: $567-580$.

Sakurai T, Yada N, Watanabe T, Arizumi T, Hagiwara S, Ueshima K, Nishida N, Fujita J, Kudo M. 2015. Cold-inducible RNAbinding protein promotes the development of liver cancer. Cancer Sci 106: 352-358.

Schibler U, Gotic I, Saini C, Gos P, Curie T, Emmenegger Y, Sinturel F, Gosselin P, Gerber A, Fleury-Olela F, et al. 2015. Clock-talk: interactions between central and peripheral circadian oscillators in mammals. Cold Spring Harb Symp Quant Biol 80: 223-232.

Shalgi R, Hurt JA, Lindquist S, Burge CB. 2014. Widespread inhibition of posttranscriptional splicing shapes the cellular transcriptome following heat shock. Cell Rep 7: 1362-1370.

Sumitomo Y, Higashitsuji H, Liu Y, Fujita T, Sakurai T, Candeias MM, Itoh K, Chiba T, Fujita J. 2012. Identification of a novel enhancer that binds Sp1 and contributes to induction of cold-inducible RNA-binding protein (cirp) expression in mammalian cells. BMC Biotechnol 12: 72.

Tamaru T, Hattori M, Honda K, Benjamin I, Ozawa T, Takamatsu K. 2011. Synchronization of circadian Per2 rhythms and HSF1-BMAL1:CLOCK interaction in mouse fibroblasts after short-term heat shock pulse. PLoS One 6: e24521.

Tattersall GJ, Sinclair BJ, Withers PC, Fields PA, Seebacher F, Cooper CE, Maloney SK. 2012. Coping with thermal challenges: physiological adaptations to environmental temperatures. Compr Physiol 2: 2151-2202.

Wang M, Marin A. 2006. Characterization and prediction of alternative splice sites. Gene 366: 219-227.

Wang M, Zhang H, Heng X, Pang Q, Sun A. 2015. Expression of cold-inducible RNA-binding protein (CIRP) in pituitary adenoma and its relationships with tumor recurrence. Med Sci Monit 21: 1256-1260.

Yang C, Carrier F. 2001. The UV-inducible RNA-binding protein A18 (A18 hnRNP) plays a protective role in the genotoxic stress response. J Biol Chem 276: 47277-47284. 


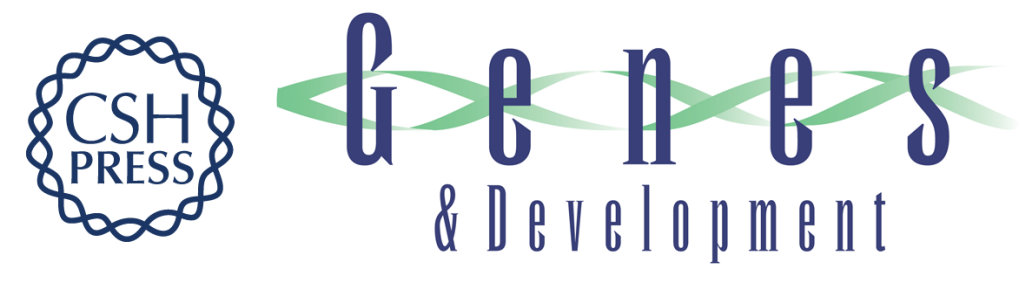

\section{Temperature regulates splicing efficiency of the cold-inducible RNA-binding protein gene Cirbp}

Ivana Gotic, Saeed Omidi, Fabienne Fleury-Olela, et al.

Genes Dev. 2016, 30: originally published online September 15, 2016

Access the most recent version at doi:10.1101/gad.287094.116

\section{Supplemental http://genesdev.cshlp.org/content/suppl/2016/09/15/gad.287094.116.DC1 \\ Material}

Related Content

Cold cuts added to the circadian smorgasbord of regulatory mechanisms Carla B. Green

Genes Dev. September , 2016 30: 1909-1910

References This article cites 48 articles, 14 of which can be accessed free at:

http://genesdev.cshlp.org/content/30/17/2005.full.html\#ref-list-1

Articles cited in:

http://genesdev.cshlp.org/content/30/17/2005.full.html\#related-urls

Creative This article, published in Genes \& Development, is available under a Creative Commons

Commons

License

License (Attribution-NonCommercial 4.0 International), as described at

http://creativecommons.org/licenses/by-nc/4.0/.

Email Alerting

Receive free email alerts when new articles cite this article - sign up in the box at the top

Service

right corner of the article or click here.

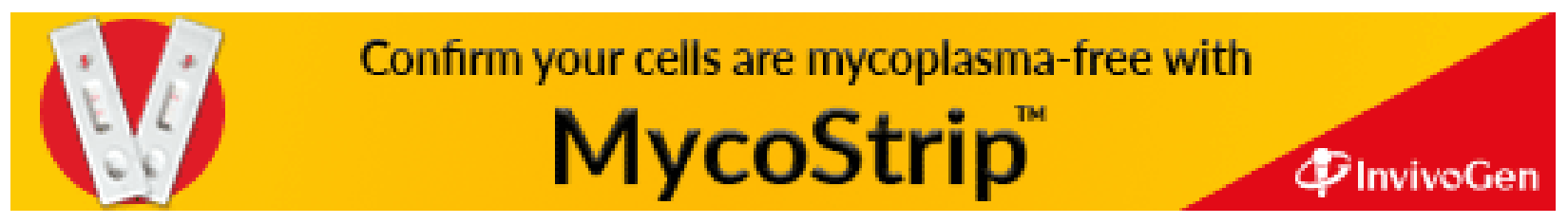

